\title{
Density Fluctuations across the Superfluid-Supersolid Phase Transition in a Dipolar Quantum Gas
}

\author{
J. Hertkorn $\odot,{ }^{1,}{ }^{*}$ J.-N. Schmidt, ${ }^{1,}{ }^{*}$ F. Böttcher $\odot,{ }^{1}$ M. Guo, ${ }^{1}$ M. Schmidt, ${ }^{1}$ K. S. H. Ng, ${ }^{1}$ S. D. Graham, ${ }^{1}$ \\ H. P. Büchler, ${ }^{2}$ T. Langen $\odot,{ }^{1}$ M. Zwierlein, ${ }^{3}$ and T. Pfau ${ }^{1, \dagger}$ \\ 15. Physikalisches Institut and Center for Integrated Quantum Science and Technology, \\ Universität Stuttgart, Pfaffenwaldring 57, 70569 Stuttgart, Germany \\ ${ }^{2}$ Institute for Theoretical Physics III and Center for Integrated Quantum Science and Technology, \\ Universität Stuttgart, Pfaffenwaldring 57, 70569 Stuttgart, Germany \\ ${ }^{3}$ MIT-Harvard Center for Ultracold Atoms, Research Laboratory of Electronics, \\ and Department of Physics, Massachusetts Institute of Technology, \\ Cambridge, Massachusetts 02139, USA
}

(Received 15 October 2020; accepted 8 January 2021; published 23 February 2021)

\begin{abstract}
Phase transitions share the universal feature of enhanced fluctuations near the transition point. Here, we show that density fluctuations reveal how a Bose-Einstein condensate of dipolar atoms spontaneously breaks its translation symmetry and enters the supersolid state of matter-a phase that combines superfluidity with crystalline order. We report on the first direct in situ measurement of density fluctuations across the superfluid-supersolid phase transition. This measurement allows us to introduce a general and straightforward way to extract the static structure factor, estimate the spectrum of elementary excitations, and image the dominant fluctuation patterns. We observe a strong response in the static structure factor and infer a distinct roton minimum in the dispersion relation. Furthermore, we show that the characteristic fluctuations correspond to elementary excitations such as the roton modes, which are theoretically predicted to be dominant at the quantum critical point, and that the supersolid state supports both superfluid as well as crystal phonons.
\end{abstract}

DOI: 10.1103/PhysRevX.11.011037

Fluctuations play a central role in quantum many-body systems. They connect the response and correlation of the system to its excitation spectrum, instabilities, phase transitions, and thermodynamic properties. A quantity that is fundamental to the theoretical description of fluctuations in many-body systems is the structure factor, which can be formulated as the Fourier transform of the density-density correlation function [1,2]. Superfluid helium is an important example of a quantum many-body state, where the determination of the structure factor is crucial to understand its elementary excitations and, therefore, improves our understanding of superfluidity [2-5]. In the case of quantum gases, the structure factor of Bose-Einstein condensates (BECs) and superfluid Fermi gases is often

\footnotetext{
*These authors contributed equally to this work.

†.pfau@physik.uni-stuttgart.de
}

Published by the American Physical Society under the terms of the Creative Commons Attribution 4.0 International license. Further distribution of this work must maintain attribution to the author(s) and the published article's title, journal citation, and DOI.
Subject Areas: Atomic and Molecular Physics, Condensed Matter Physics, Quantum Physics

investigated by Bragg spectroscopy [6-8]. In contactinteracting BECs, this technique enables the study of the spectrum and collective modes [9]. In dipolar BECs, it provides indications of the roton minimum in the dispersion relation $[10,11]$ analogous to the neutron and $\mathrm{x}$-ray scattering data for helium [2,12]. A different approach is to look at the condensate density directly in situ, which provides access to finite temperature and quantum fluctuations [13-19] and enables one to extract the static structure factor simultaneously at all momenta.

The roton minimum in both helium and dipolar quantum gases is accompanied by a characteristic peak in the static structure factor close to the roton momentum [2,20-22]. Unlike in helium, however, the contact interactions in dipolar quantum gases are tunable [23]. This tunability allows for precise control of the dispersion relation and the controllable softening of the roton minimum. The roton modes associated to this minimum manifest as density modulations on top of the ground-state density distribution [24,25]. An instability in the ground state can appear once the roton minimum is sufficiently soft. Since these modes represent precursors to solidification, dipolar BECs have long been proposed as candidates for the elusive supersolid 
state of matter, which simultaneously combines crystalline order with superfluidity [26].

Recently, a dipolar supersolid state of matter has been realized through a phase transition from a BEC to an array of coherent quantum droplets [27-33] by precisely tuning the contact interaction strength. Close to the transition point, these droplets are immersed in a superfluid background, and, by lowering the scattering length further, the superfluid fraction decreases toward a regime of isolated droplets. As the superfluid-supersolid phase transition is governed by intrinsic interactions, it is of interest to study the fluctuations that emerge across the transition [22,34,35], facilitate structure formation [16,21,36-39], and give rise to the supersolid state. The low-lying collective modes are shown to be particularly interesting regarding the aspect of supersolidity in this system [30-32,40]. Those modes are facilitated by a continuous superfluidity across the droplet array, despite the translational symmetry breaking.

Here, we provide the first direct in situ observation of density fluctuations across the superfluid-supersolid phase transition in a trapped dipolar quantum gas. By analyzing hundreds of in situ images of the atomic cloud around the phase transition point, we spatially resolve characteristic fluctuation patterns that arise across the transition. From the observed fluctuations, we determine the static structure factor and estimate the spectrum of elementary excitations. We observe a strong peak in the static structure factor and an associated roton minimum in the dispersion relation. Moreover, we experimentally determine that the dominant fluctuations at the transition point correspond to two degenerate roton modes [30,40] and that the supersolid state supports both superfluid as well as crystal phonons in a narrow range of scattering lengths. Our study combines the fluctuations with the excitation spectrum of a dipolar supersolid and highlights its bipartite nature between the superfluid BEC and the crystalline isolated droplets.

The rotonic dispersion relation of strongly dipolar BECs $[11,24,25]$ is schematically shown in Fig. 1(a). The system becomes more susceptible to density fluctuations as the roton minimum softens. These density fluctuations are associated with a characteristic peak $[2,41-44]$ in the static structure factor, illustrated in Fig. 1(b). This peak can be understood by considering the general Feynman-Bijl formula $S(\boldsymbol{k})=\hbar^{2} \boldsymbol{k}^{2} / 2 m \varepsilon(\boldsymbol{k})$ [2,3], connecting the static structure factor $S(\boldsymbol{k})$ to the excitation spectrum $\epsilon(\boldsymbol{k})$ at zero temperature. As the energy of the roton modes drops near zero, the density fluctuations and, thus, the structure factor increase dramatically. Eventually, the roton minimum sufficiently softens in order for the system to enter the roton instability. This roton instability triggers the phase transition to a dipolar supersolid and arrays of isolated quantum droplets.

To study the static structure factor experimentally, we prepare a dipolar BEC with typically $40 \times 10^{3}{ }^{162} \mathrm{Dy}$ atoms at
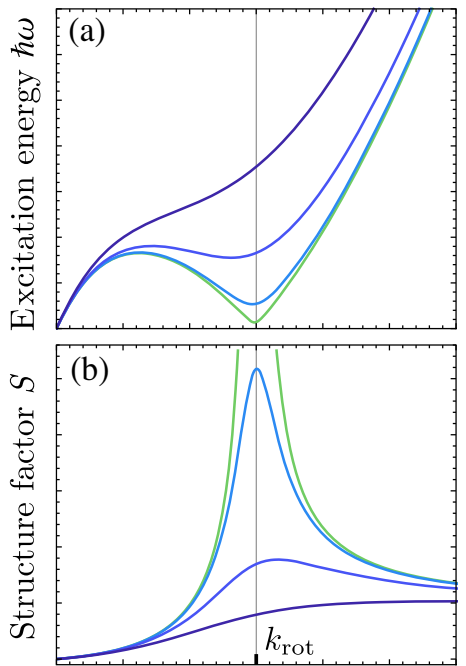

Momentum $k$

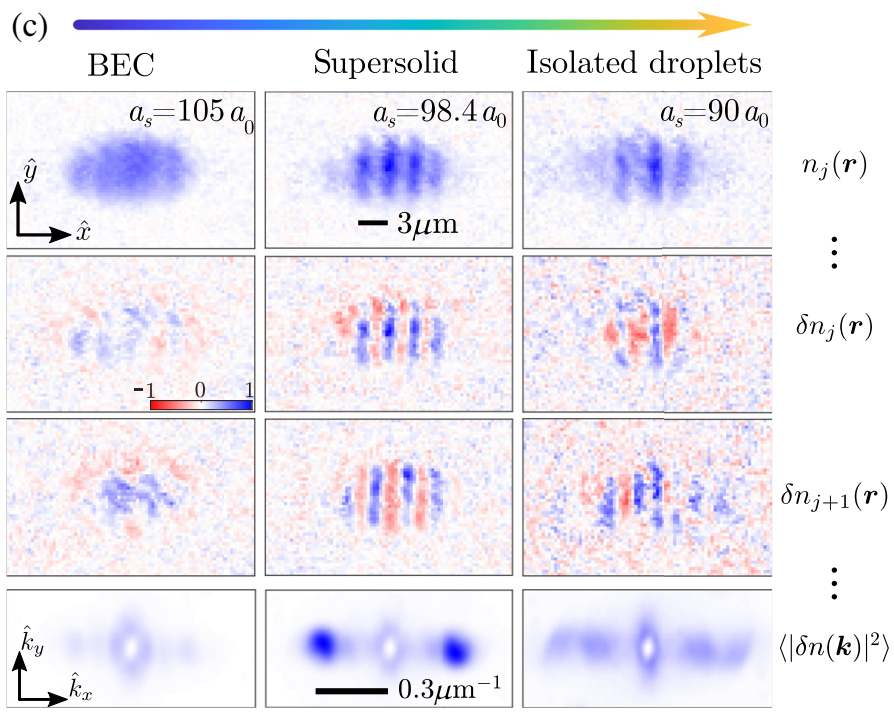

FIG. 1. (a),(b) Schematic of the dispersion relation $\hbar \omega(k)$ and associated static structure factor $S(k)$ of an elongated and strongly dipolar BEC [22]. A decrease in scattering length $a_{s}$ causes a roton minimum to emerge in the excitation spectrum, associated with a characteristic peak in the static structure factor. The roton momentum $k_{\text {rot }}$ is indicated where the roton minimum drops near zero. (c) For a given scattering length $a_{s}$, we observe a large number of in situ densities $n_{j}(\boldsymbol{r})$ and calculate their mean $\langle n(\boldsymbol{r})\rangle$ and the density fluctuations $\delta n_{j}(\boldsymbol{r})=n_{j}(\boldsymbol{r})-\langle n(\boldsymbol{r})\rangle$ as the deviation of the in situ images from their mean. Investigating the mean power spectrum of the fluctuations $\left\langle|\delta n(\boldsymbol{k})|^{2}\right\rangle$ for different scattering lengths across the transition allows us to directly observe the static structure factor as the system passes from BEC to supersolid to isolated droplet states. The colored arrow on top indicates the direction toward lower scattering lengths, passing from BEC to supersolid to isolated droplet regimes. The color map used for the images shows the normalized amplitude of densities, density fluctuations, and mean power spectra, respectively, with the color bar referring to each row separately. 
temperatures of approximately $20 \mathrm{nK}$ in a cigar-shaped trap with trapping frequencies $\omega / 2 \pi=[30(1), 89(2), 108(2)] \mathrm{Hz}$ and a magnetic field oriented along $\hat{\boldsymbol{y}}$ [45]. The scattering length is tuned via a double Feshbach resonance [46] to final values between $90 a_{0}$ and $105 a_{0}$ by linearly ramping the magnetic field in $30 \mathrm{~ms}$. We wait for $15 \mathrm{~ms}$ to allow for the system to equilibrate, and then the atoms are imaged using phase-contrast imaging along the $\hat{z}$ axis with a resolution of approximately $1 \mu \mathrm{m}$. We find either a BEC, a supersolid phase (SSP), or isolated droplets (IDs) for large $\left(a_{s} \simeq 105 a_{0}\right)$, intermediate $\left(a_{s} \simeq 98.4 a_{0}\right)$, and small $\left(a_{s} \simeq 90 a_{0}\right)$ scattering lengths, respectively [30]. We accumulate enough averages for a statistical evaluation of the structure factor by repeating the experiment around 200 times for every scattering length.

We obtain $S(\boldsymbol{k})$ experimentally by analyzing the in situ images as illustrated in Fig. 1(c). For every scattering length, we center the in situ densities $n_{j}(\boldsymbol{r})$ to their center of mass and normalize them to the mean atom number. With the former step we remove contributions of the dipole center of mass motion [30], and with the latter we correct for shot to shot total atom number fluctuations $[14,45]$ that otherwise give contributions to $S(\boldsymbol{k})$ near $\boldsymbol{k}=0$. From these in situ images, we obtain the mean image $\langle n(\boldsymbol{r})\rangle$ and the density fluctuations $\delta n_{j}(\boldsymbol{r})=n_{j}(\boldsymbol{r})-\langle n(\boldsymbol{r})\rangle$ as the deviation of the in situ images from their mean. With the Fourier transform of the density fluctuation $\delta n_{j}(\boldsymbol{k})=\int d^{3} r \delta n_{j}(\boldsymbol{r}) e^{i \boldsymbol{k} \cdot \boldsymbol{r}}$ we obtain the mean power spectrum of the fluctuations $\left\langle|\delta n(\boldsymbol{k})|^{2}\right\rangle$. In homogeneous systems, the static structure factor can be directly written as $S(\boldsymbol{k})=\left\langle|\delta n(\boldsymbol{k})|^{2}\right\rangle / N$, where $N$ is the atom number $[2,16,18]$. In practice, the interpretation is less straightforward [19,47-49] since the expectation values of the density $\langle n(\boldsymbol{r})\rangle$ are spatially dependent due to the finite size and the translational symmetry breaking in the supersolid and droplet regime. Nonetheless $S(\boldsymbol{k})$ gives insight into the strength of fluctuations [14,19,50-52] and is a quantity that can be continuously evaluated from the BEC via the supersolid to the isolated droplet regime. We note that our evaluation is limited to intermediate momenta between $k_{\min } / 2 \pi \simeq 0.08 \mu \mathrm{m}^{-1}$ and $k_{\max } / 2 \pi \simeq 1 \mu \mathrm{m}^{-1}$ due to the finite system size and the finite resolution of our imaging system, respectively $[16,19]$. We extract the static structure factor $S\left(k_{x}, k_{y}, k_{z}=0\right)$ cut along the $k_{z}=0$ plane according to the Fourier slice theorem, since the atomic densities are integrated along the line of sight during the imaging process $[45,53]$. Because of the cigar-shaped trap geometry, the fluctuations predominantly show structure along $\hat{k}_{x}$ [see Fig. 1(c)], which allows us to extract a cut of the mean power spectrum at $k_{y}=0$ to obtain the 1D structure factor $S\left(k_{x}\right)$.

Using the above described analysis, we obtain $S\left(k_{x}\right)$ across the phase transition as shown in Fig. 2. In the BEC regime at $a_{s} \simeq 104 a_{0}$, we find the structure factor to be

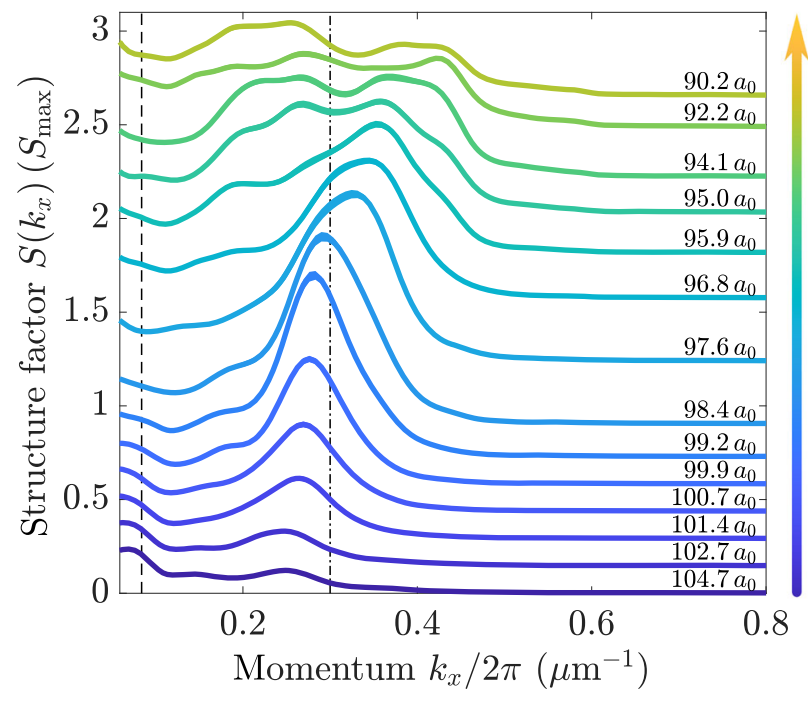

FIG. 2. Experimentally determined structure factor in units of $S_{\max }=260$ for different scattering lengths $a_{s}$. The dashed line on the left indicates the smallest momentum $k_{\min } / 2 \pi \simeq 0.08 \mu \mathrm{m}^{-1}$ available due to the finite size of the system along the $x$ direction. The dash-dotted line at $k_{x} / 2 \pi \simeq 0.3 \mu \mathrm{m}^{-1}$ roughly indicates the inverse droplet spacing in the droplet regime, which coincides with the roton momentum at the transition point. Errors are indicated as increased thickness of the lines and are obtained by the bootstrapping method $[45,54]$. For illustration purposes, the lines for smaller $a_{s}$ are shifted up. The momentum axis is sampled with a spacing of $\Delta k_{x} / 2 \pi \simeq 0.007 \mu \mathrm{m}^{-1}$. The colored arrow indicates the direction toward lower scattering lengths, passing from BEC to supersolid to isolated droplet regimes.

relatively flat with the exception of a small peak at around $k_{x} / 2 \pi \simeq 0.25 \mu \mathrm{m}^{-1}$. This peak is an indication that, far in the BEC regime, roton modes can be excited [30] and, consequently, that the spectrum features modifications from a purely contact interacting quantum gas.

As the scattering length is reduced, the position and amplitude of this characteristic peak are observed to increase continuously toward the phase transition point $\left(a_{s} \simeq 98.4 a_{0}\right)$. A continuously growing peak amplitude of the structure factor signals enhanced fluctuations, consistent with a softening roton minimum toward the transition point. The structure factor reaches its maximum value as a function of the scattering length at the transition point and is located at the roton momentum $k_{\text {rot }} / 2 \pi \simeq 0.29 \mu \mathrm{m}^{-1}$. This value is mainly given by the harmonic oscillator length $l_{y}$ along the magnetic field direction [55]. At the transition point, the enhanced fluctuations provide the roton instability and lead to the formation of supersolid quantum droplets, whose spacing $d \simeq 3 \mu \mathrm{m}$ smoothly matches the roton wavelength $2 \pi / k_{\text {rot }}[28,30]$. The observed increase of the roton momentum toward smaller scattering lengths can be understood in a variational approach of elongated dipolar condensates [56]. Around the transition point, the in situ densities we observe from shot to shot show droplets immersed in an overall BEC background, 
constituting the supersolid state of matter [27-33,40,57]. Here, the density fluctuation patterns become most clear and show spatial oscillations [see Fig. 1(c), middle column]. These characteristic fluctuations can directly be attributed to the symmetric and antisymmetric roton modes we find in our previous work [40].

After crossing the phase transition $\left(a_{s} \lesssim 98.4 a_{0}\right)$, the peak amplitude of the structure factor decreases, and a shoulder develops at smaller momenta. This shoulder increases further for smaller scattering lengths and eventually leads to a double-peak structure as seen in Fig. 2. The origin of this rising double peak can be understood by means of a principal component analysis.

The maximum of the structure factor for different scattering lengths acts as a measure of the density fluctuation strength across the superfluid to supersolid phase transition. It quickly increases from the BEC side when approaching the phase transition, indicating a significant enhancement of the characteristic fluctuations close to the phase transition point. We see that the increase from the BEC side toward the phase transition is sharper than the decrease on the droplet side. The magnitude of the structure factor $\left(S_{\max }=260\right)$ can mainly be explained by thermal enhancement of the participating low-energy modes.

To estimate the dispersion relation based on the experimentally determined structure factor, we use the relation

$$
S(\boldsymbol{k})=\frac{\hbar^{2} \boldsymbol{k}^{2}}{2 m \varepsilon(\boldsymbol{k})} \operatorname{coth}\left(\frac{\varepsilon(\boldsymbol{k})}{2 k_{B} T}\right),
$$

which extends the Feynman-Bijl formula $S(\boldsymbol{k})=\hbar^{2} \boldsymbol{k}^{2} / 2 m \varepsilon(\boldsymbol{k})$, valid at $T=0$, to nonzero temperatures $T[2,16,20]$. At nonzero temperatures and small excitation energies, the contribution of low-lying modes to the structure factor can easily be enhanced by several orders of magnitude. Close to the transition point, where the roton gap $\Delta_{\text {rot }}$ is small compared to the temperature of the system $\left(\hbar \Delta_{\text {rot }} / k_{B} T \lesssim 1\right)$, Eq. (1) can be expanded, and the peak of the static structure factor scales as $S_{\max } \sim T / \Delta_{\text {rot }}^{2}[20]$. A rising temperature, therefore, enhances the population of low-lying modes and leads to an increase of the measured peak in the static structure factor [45]. Note that Eq. (1) is an excellent description of the structure factor for a weakly interacting superfluid, where the excitation spectrum is dominated by a single mode and where the influence of the quantum as well as thermal depletion can be ignored. Although we study a finite system, leading to a discrete excitation spectrum, a continuous approximation to the dispersion relation yields a meaningful estimate for the excitation energies [45]. We show the resulting spectrum $\omega\left(k_{x}\right) / 2 \pi=\varepsilon\left(k_{x}\right) / h$ in Fig. 3 . To do so, we assume a mean temperature of $20 \mathrm{nK}$, a conservative approximation to include additional minor heating during the preparation [45]. In Fig. 3, one can see a small roton minimum already well above the trap

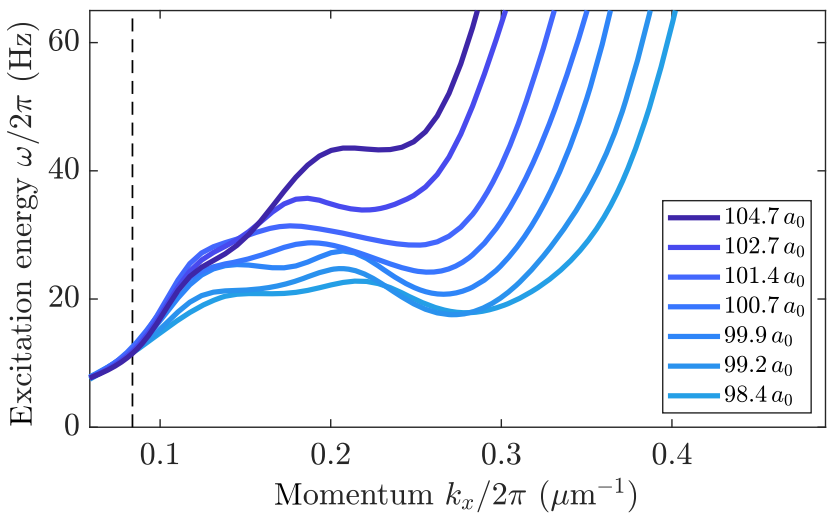

FIG. 3. Experimentally determined excitation energy $\omega\left(k_{x}\right)$ according to Eq. (1) assuming a temperature of $20 \mathrm{nK}$, for scattering lengths above the phase transition point. A clear roton minimum at finite momentum is observed that softens toward the transition point.

frequency of $30 \mathrm{~Hz}$ for a large scattering length. The roton minimum softens and moves toward higher momenta $k_{x}$ as the scattering length is lowered and finally reaches its lowest energy at the phase transition point. After crossing the phase transition point, when the crystalline structure has developed, the excitation spectrum should have a band structure due to the translational symmetry breaking. In this case, Eq. (1) is no longer necessarily justified, as several modes contribute to the excitation spectrum, and, therefore, it is no longer straightforward to extract the excitation spectrum from the measured static structure factor.

To gain a better insight into the modes that dominantly contribute to the fluctuations, we use principal component analysis (PCA) [58] on the density fluctuations for all scattering lengths combined. This model-free statistical analysis is a general method to extract dominant components or to reduce the dimensionality of a dataset. We study the principal components (PCs) across the phase transition, since there is a direct correspondence [59] to the dominant collective excitations obtained with the Bogoliubov-de Gennes (BdG) formalism [40]. This study allows us to identify and compare the most dominant PCs with specific BdG modes and study how their weight behaves across the transition, as shown in Figs. 4 and 5.

The first principal component is structureless and represents only the global atom number fluctuation $[59,60]$. The subsequent principal components are shown in Figs. 4(a) and 4(b). These two components are dominant across the phase transition and represent a periodic spatial pattern. Close to the transition point, we can identify these characteristic patterns in many single-shot realizations of the density fluctuations, as shown in the central column in Fig. 1(c). We compare the profiles of these PCs to the antisymmetric and symmetric roton modes from the BdG theory at the transition point in Figs. 4(c) and 4(d) and find them to be in excellent agreement. These two roton modes are developing into the 

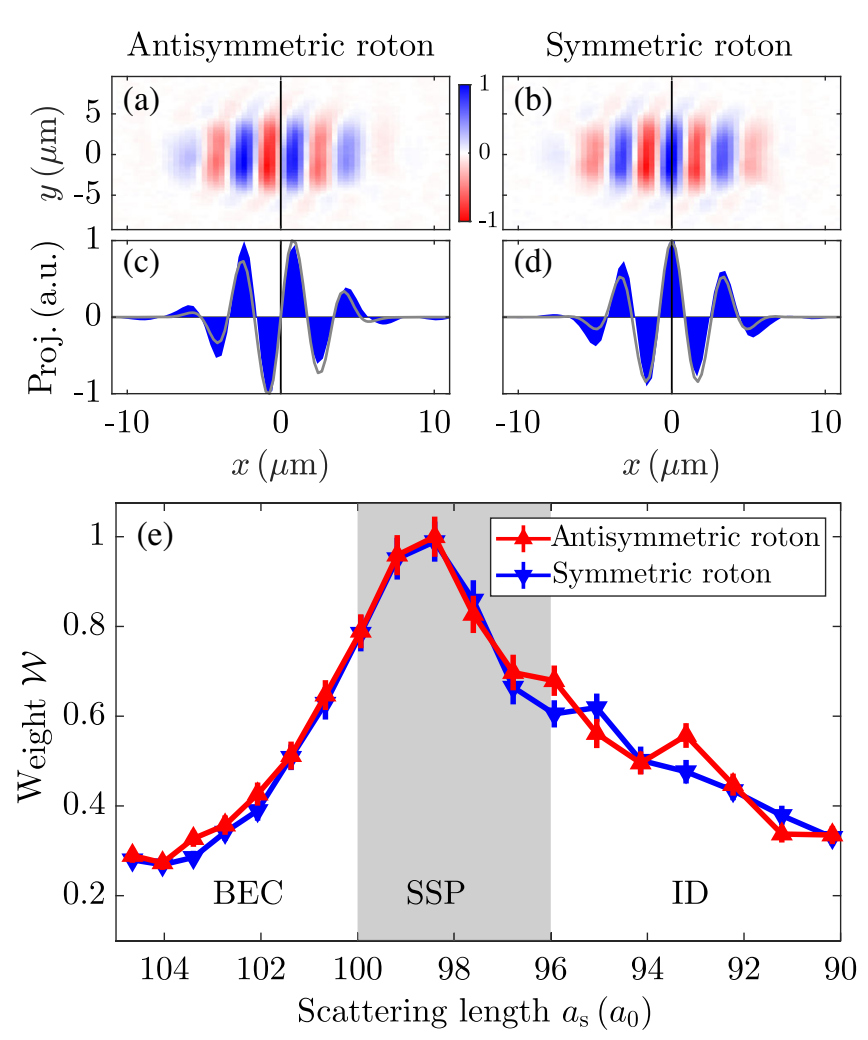

FIG. 4. (a),(b) Spatial structure of two principal components with the largest weight dominating the experimental dataset. (c), (d) Projections of (a),(b) onto the $x$ axis (blue), with a comparison to the antisymmetric and symmetric roton density fluctuations from our BdG calculation [40] (gray). (e) Mean absolute weight $\mathcal{W}$ of the symmetric and antisymmetric roton, normalized to the weight of the antisymmetric roton mode at the transition point, which is the maximum weight of all principal components over the whole scattering length range. The roton modes are dominant, and their weights are almost degenerate from the BEC $\left(a_{s}=104.7 a_{0}\right)$ leading up to the transition point $\left(a_{s}=98.4 a_{0}\right)$. The gray area indicates the supersolid region previously determined [30]. Error bars indicate the standard error of the mean.

Goldstone [30] and amplitude (Higgs) [40] modes of the supersolid.

The mean absolute value of the weights for these two PCs are shown in Fig. 4(e) as an indication of their strength across the phase transition. Starting from the BEC side, where they are comparable in strength to other modes, these PCs gain rapidly in strength as the phase transition is approached [45]. We note that the maximum of the structure factor behaves similarly to the weights of these two PCs as a function of the scattering length. Leading up to the quantum critical point at $a_{s} \simeq 98.4 a_{0}$, these two modes have identical weights, in accordance to our previous work [40], in which we show that the two roton modes remain degenerate while softening toward the phase transition.

Further into the isolated droplet regime, the weight of the roton PCs decreases and other PCs become more important, because further modes are softening. In Fig. 5, we present

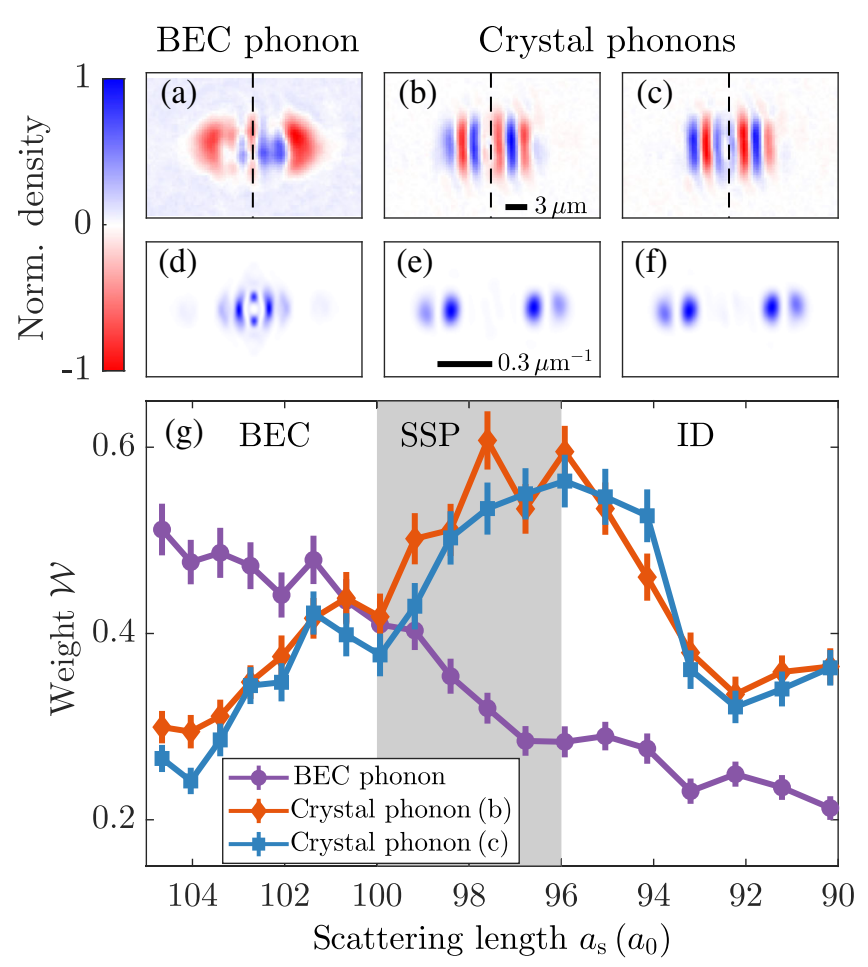

FIG. 5. Higher principal components corresponding to the phonons of the crystal and the BEC (a)-(c) including their Fourier transforms (d)-(f) and weights (g). (a) Quadrupole mode of the BEC. The antisymmetric (b) and the symmetric (c) breathing mode of the crystal show a clear splitting in their Fourier transform (e),(f). (g) Comparison of the mean absolute weights $\mathcal{W}$ of these modes featuring a clear overlap region that indicates a supersolid phase. The gray shaded area shows the previously determined supersolid region [30]. Error bars indicate the standard error of the mean. The weights are normalized to the weight of the antisymmetric roton mode at the transition point, which is the maximal weight of all principal components over the whole scattering length range.

the three next higher PCs that correspond to the BEC phonon (a) and the antisymmetric (b) and symmetric (c) crystal phonon, respectively. The quadrupole mode of the BEC has a relatively high weight in the BEC regime and vanishes for small scattering lengths toward the isolated droplet regime. The breathing or lowest phonon modes of the droplet crystal differ in their symmetry with respect to the center of mass indicated by the vertical dashed line. They show a clear splitting in the Fourier transform [Figs. 5(d) and 5(e)], explaining the observed double-peak structure in $S\left(k_{x}\right)$ for low scattering lengths. This splitting can be understood as the appearance of the band structure, where excitations are split around the edge of the Brillouin zone. These modes have an appreciable weight for low scattering length only after crossing the phase transition [Fig. 5(g)]. In the experiment, the excitation of the crystal breathing mode is further enhanced by the preparation process $[28,31]$. Note that there is a small region close to the phase transition where both types of modes have a 
nonvanishing weight. This subtle feature shows the coexistence of both BEC and droplet crystal, which is a prerequisite of the supersolid nature of the phase. One can see that the supersolid state supports both types of excitations - the phonon of the superfluid BEC and the crystalline phonons of the droplets.

In conclusion, we report the first in situ measurement of the density fluctuations across the superfluid to supersolid phase transition in a dipolar quantum gas. We quantify the fluctuation strength across the transition by the static structure factor $S\left(k_{x}\right)$ using a statistical evaluation of in situ images and find a characteristic peak in $S\left(k_{x}\right)$ that strongly increases toward the phase transition point. We show that this peak is unambiguously dominated by the low-lying modes of the rotonic dispersion relation. The characteristic fluctuations close to the transition point are stronger compared to the BEC or isolated droplet regime. The large amplitude of the measured static structure factor reveals the important role played by temperature at the phase transition, an aspect which has so far been absent in the discussion of the superfluid-supersolid phase transition. Using principal component analysis, we spatially resolve the dominant fluctuations and identify them as two roton modes. Furthermore, we show that the supersolid supports both superfluid and crystal phonons. Our study provides a promising outlook to extract thermodynamic properties [21] and possibly universal access to the condensate fraction [39] of the supersolid state. Exciting avenues for future work include using fluctuations as a tool for thermometry of the supersolid state [61], understanding the out of equilibrium dynamics that arise when crossing the phase transition [62,63], and exploring the roles of fluctuations in the Kibble-Zurek mechanism [34,35,64].

We thank Tobias Ilg and Jan Kumlin for valuable discussions. M. G. and M.Z. acknowledge funding from the Alexander von Humboldt Foundation. This work is supported by the German Research Foundation (DFG) within FOR2247 under Pf381/16-1 and Bu2247/1, Pf381/ 20-1, FUGG INST41/1056-1, and the QUANT:ERA collaborative project MAQS.

[1] A. Griffin, Excitations in a Bose-Condensed Liquid, Cambridge Studies in Low Temperature Physics (Cambridge University Press, Cambridge, England, 1993).

[2] L. P. Pitaevskii and S. Stringari, Bose-Einstein Condensation and Superfluidity, International Series of Monographs on Physics (Oxford University Press, New York, 2016).

[3] R. P. Feynman, Atomic Theory of the Two-Fluid Model of Liquid Helium, Phys. Rev. 94, 262 (1954).

[4] J. A. Tarvin, F. Vidal, and T. J. Greytak, Measurements of the Dynamic Structure Factor near the Lambda Temperature in Liquid Helium, Phys. Rev. B 15, 4193 (1977).

[5] S. Balibar, The Discovery of Superfluidity, J. Low Temp. Phys. 146, 441 (2007).
[6] J. Stenger, S. Inouye, A. P. Chikkatur, D. M. Stamper-Kurn, D. E. Pritchard, and W. Ketterle, Bragg Spectroscopy of a Bose-Einstein Condensate, Phys. Rev. Lett. 82, 4569 (1999).

[7] G. Veeravalli, E. Kuhnle, P. Dyke, and C. J. Vale, Bragg Spectroscopy of a Strongly Interacting Fermi Gas, Phys. Rev. Lett. 101, 250403 (2008).

[8] E. D. Kuhnle, H. Hu, X.-J. Liu, P. Dyke, M. Mark, P. D. Drummond, P. Hannaford, and C. J. Vale, Universal Behavior of Pair Correlations in a Strongly Interacting Fermi Gas, Phys. Rev. Lett. 105, 070402 (2010).

[9] D. M. Stamper-Kurn, A. P. Chikkatur, A. Görlitz, S. Inouye, S. Gupta, D. E. Pritchard, and W. Ketterle, Excitation of Phonons in a Bose-Einstein Condensate by Light Scattering, Phys. Rev. Lett. 83, 2876 (1999).

[10] G. Bismut, B. Laburthe-Tolra, E. Maréchal, P. Pedri, O. Gorceix, and L. Vernac, Anisotropic Excitation Spectrum of a Dipolar Quantum Bose Gas, Phys. Rev. Lett. 109, 155302 (2012).

[11] D. Petter, G. Natale, R. M. W. van Bijnen, A. Patscheider, M. J. Mark, L. Chomaz, and F. Ferlaino, Probing the Roton Excitation Spectrum of a Stable Dipolar Bose Gas, Phys. Rev. Lett. 122, 183401 (2019).

[12] D. M. Ceperley, Path Integrals in the Theory of Condensed Helium, Rev. Mod. Phys. 67, 279 (1995).

[13] S. Folling, F. Gerbier, A. Widera, O. Mandel, T. Gericke, and I. Bloch, Spatial Quantum Noise Interferometry in Expanding Ultracold Atom Clouds, Nature (London) 434, 481 (2005).

[14] J. Esteve, J.-B. Trebbia, T. Schumm, A. Aspect, C. I. Westbrook, and I. Bouchoule, Observations of Density Fluctuations in an Elongated Bose Gas: Ideal Gas and Quasicondensate Regimes, Phys. Rev. Lett. 96, 130403 (2006).

[15] N. Gemelke, X. Zhang, H. Chen-Lung, and C. Chin, In Situ Observation of Incompressible Mott-Insulating Domains in Ultracold Atomic Gases, Nature (London) 460, 995 (2009).

[16] C.-L. Hung, X. Zhang, L.-C. Ha, S.-K. Tung, N. Gemelke, and C. Chin, Extracting Density-Density Correlations from In Situ Images of Atomic Quantum Gases, New J. Phys. 13, 075019 (2011).

[17] C.-L. Hung, X. Zhang, N. Gemelke, and C. Chin, Observation of Scale Invariance and Universality in Two-Dimensional Bose Gases, Nature (London) 470, 236 (2011).

[18] C.-L. Hung, V. Gurarie, and C. Chin, From Cosmology to Cold Atoms: Observation of Sakharov Oscillations in a Quenched Atomic Superfluid, Science 341, 1213 (2013).

[19] M. Schemmer, A. Johnson, and I. Bouchoule, Monitoring Squeezed Collective Modes of a One-Dimensional Bose Gas after an Interaction Quench Using Density-Ripple Analysis, Phys. Rev. A 98, 043604 (2018).

[20] M. Klawunn, A. Recati, L. P. Pitaevskii, and S. Stringari, Local Atom-Number Fluctuations in Quantum Gases at Finite Temperature, Phys. Rev. A 84, 033612 (2011).

[21] J. Hofmann and W. Zwerger, Exact Relations for Dipolar Quantum Gases, arXiv:2007.13774.

[22] S. Pal, D. Baillie, and P. B. Blakie, Excitations and Number Fluctuations in an Elongated Dipolar Bose-Einstein Condensate, Phys. Rev. A 102, 043306 (2020). 
[23] C. Chin, R. Grimm, P. Julienne, and E. Tiesinga, Feshbach Resonances in Ultracold Gases, Rev. Mod. Phys. 82, 1225 (2010).

[24] L. Santos, G. V. Shlyapnikov, and M. Lewenstein, RotonMaxon Spectrum and Stability of Trapped Dipolar BoseEinstein Condensates, Phys. Rev. Lett. 90, 250403 (2003).

[25] S. Giovanazzi and D. H. J. O'Dell, Instabilities and the Roton Spectrum of a Quasi-1D Bose-Einstein Condensed Gas with Dipole-Dipole Interactions, Eur. Phys. J. D 31, 439 (2004).

[26] M. Boninsegni and N. V. Prokof'ev, Colloquium: Supersolids: What and Where Are They?, Rev. Mod. Phys. 84, 759 (2012).

[27] L. Tanzi, E. Lucioni, F. Famà, J. Catani, A. Fioretti, C. Gabbanini, R. N. Bisset, L. Santos, and G. Modugno, Observation of a Dipolar Quantum Gas with Metastable Supersolid Properties, Phys. Rev. Lett. 122, 130405 (2019).

[28] F. Böttcher, J.-N. Schmidt, M. Wenzel, J. Hertkorn, M. Guo, T. Langen, and T. Pfau, Transient Supersolid Properties in an Array of Dipolar Quantum Droplets, Phys. Rev. X 9, 011051 (2019).

[29] L. Chomaz, D. Petter, P. Ilzhöfer, G. Natale, A. Trautmann, C. Politi, G. Durastante, R. M. W. van Bijnen, A. Patscheider, M. Sohmen, M. J. Mark, and F. Ferlaino, Long-Lived and Transient Supersolid Behaviors in Dipolar Quantum Gases, Phys. Rev. X 9, 021012 (2019).

[30] M. Guo, F. Böttcher, J. Hertkorn, J.-N. Schmidt, M. Wenzel, H. P. Büchler, T. Langen, and T. Pfau, The Low-Energy Goldstone Mode in a Trapped Dipolar Supersolid, Nature (London) 574, 386 (2019).

[31] L. Tanzi, S. M. Roccuzzo, E. Lucioni, F. Fam, A. Fioretti, C. Gabbanini, G. Modugno, A. Recati, and S. Stringari, Supersolid Symmetry Breaking from Compressional Oscillations in a Dipolar Quantum Gas, Nature (London) 574, 382 (2019).

[32] G. Natale, R. M. W. van Bijnen, A. Patscheider, D. Petter, M. J. Mark, L. Chomaz, and F. Ferlaino, Excitation Spectrum of a Trapped Dipolar Supersolid and Its Experimental Evidence, Phys. Rev. Lett. 123, 050402 (2019).

[33] F. Böttcher, J.-N. Schmidt, J. Hertkorn, K. N. S. Ng, S. D. Graham, M. Guo, T. Langen, and T. Pfau, New States of Matter with Fine-Tuned Interactions: Quantum Droplets and Dipolar Supersolids, Rep. Prog. Phys. 84, 012403 (2021).

[34] W. H. Zurek, The Shards of Broken Symmetry, Nature (London) 382, 296 (1996).

[35] A. Del Campo, T. W. B. Kibble, and W. H. Zurek, Causality and Non-equilibrium Second-Order Phase Transitions in Inhomogeneous Systems, J. Phys. Condens. Matter 25, 404210 (2013).

[36] R. Mottl, F. Brennecke, K. Baumann, R. Landig, T. Donner, and T. Esslinger, Roton-Type Mode Softening in a Quantum Gas with Cavity-Mediated Long-Range Interactions, Science 336, 1570 (2012).

[37] R. Landig, F. Brennecke, R. Mottl, T. Donner, and T. Esslinger, Measuring the Dynamic Structure Factor of a Quantum Gas Undergoing a Structural Phase Transition, Nat. Commun. 6, 7046 (2015).

[38] Y. Kora and M. Boninsegni, Patterned Supersolids in Dipolar Bose Systems, J. Low Temp. Phys. 197, 337 (2019).
[39] Yu. E. Lozovik, I. L. Kurbakov, G. E. Astrakharchik, and J. Boronat, Universal Relation between the Static Structure Factor and the Condensate Fraction, arXiv:2007.16050.

[40] J. Hertkorn, F. Böttcher, M. Guo, J.-N. Schmidt, T. Langen, H. P. Büchler, and T. Pfau, Fate of the Amplitude Mode in a Trapped Dipolar Supersolid, Phys. Rev. Lett. 123, 193002 (2019).

[41] A. S. Arkhipov, G. E. Astrakharchik, A. V. Belikov, and Yu. E. Lozovik, Ground-State Properties of a OneDimensional System of Dipoles, J. Exp. Theor. Phys. Lett. 82, 39 (2005).

[42] G. E. Astrakharchik, J. Boronat, I. L. Kurbakov, and Yu. E. Lozovik, Quantum Phase Transition in a Two-Dimensional System of Dipoles, Phys. Rev. Lett. 98, 060405 (2007).

[43] P. B. Blakie, D. Baillie, and R. N. Bisset, Roton Spectroscopy in a Harmonically Trapped Dipolar Bose-Einstein Condensate, Phys. Rev. A 86, 021604(R) (2012).

[44] P. B. Blakie, D. Baillie, and R. N. Bisset, Depletion and Fluctuations of a Trapped Dipolar Bose-Einstein Condensate in the Roton Regime, Phys. Rev. A 88, 013638 (2013).

[45] See Supplemental Material at http://link.aps.org/ supplemental/10.1103/PhysRevX.11.011037 for further details on the experiment, analysis, and simulation.

[46] F. Böttcher, M. Wenzel, J.-N. Schmidt, M. Guo, T. Langen, I. Ferrier-Barbut, T. Pfau, R. Bombín, J. Sánchez-Baena, J. Boronat, and F. Mazzanti, Dilute Dipolar Quantum Droplets beyond the Extended Gross-Pitaevskii Equation, Phys. Rev. Research 1, 033088 (2019).

[47] M. Naraschewski and R. J. Glauber, Spatial Coherence and Density Correlations of Trapped Bose Gases, Phys. Rev. A 59, 4595 (1999).

[48] F. Zambelli, L. Pitaevskii, D. M. Stamper-Kurn, and S. Stringari, Dynamic Structure Factor and Momentum Distribution of a Trapped Bose Gas, Phys. Rev. A 61, 063608 (2000).

[49] J. Steinhauer, R. Ozeri, N. Katz, and N. Davidson, Peak in the Static Structure Factor of a Bose-Einstein Condensate, Phys. Rev. A 72, 023608 (2005).

[50] A. Imambekov, I. E. Mazets, D. S. Petrov, V. Gritsev, S. Manz, S. Hofferberth, T. Schumm, E. Demler, and J. Schmiedmayer, Density Ripples in Expanding Low-Dimensional Gases as a Probe of Correlations, Phys. Rev. A 80, 033604 (2009).

[51] J. Armijo, T. Jacqmin, K. V. Kheruntsyan, and I. Bouchoule, Probing Three-Body Correlations in a Quantum Gas Using the Measurement of the Third Moment of Density Fluctuations, Phys. Rev. Lett. 105, 230402 (2010).

[52] T. Jacqmin, J. Armijo, T. Berrada, K. V. Kheruntsyan, and I. Bouchoule, Sub-Poissonian Fluctuations in a 1D Bose Gas: From the Quantum Quasicondensate to the Strongly Interacting Regime, Phys. Rev. Lett. 106, 230405 (2011).

[53] A. Blumkin, S. Rinott, R. Schley, A. Berkovitz, I. Shammass, and J. Steinhauer, Observing Atom Bunching by the Fourier Slice Theorem, Phys. Rev. Lett. 110, 265301 (2013).

[54] B. Efron, Bootstrap Methods: Another Look at the Jackknife, Ann. Stat. 7, 1 (1979).

[55] L. Chomaz, R. M. W. Van Bijnen, D. Petter, G. Faraoni, S. Baier, J. H. Becher, M. J. Mark, F. Wächtler, L. Santos, and 
F. Ferlaino, Observation of Roton Mode Population in a Dipolar Quantum Gas, Nat. Phys. 14, 442 (2018).

[56] P. B. Blakie, D. Baillie, and S. Pal, Variational Theory for the Ground State and Collective Excitations of an Elongated Dipolar Condensate, Commun. Theor. Phys. 72, 085501 (2020).

[57] S. M. Roccuzzo and F. Ancilotto, Supersolid Behavior of a Dipolar Bose-Einstein Condensate Confined in a Tube, Phys. Rev. A 99, 041601(R) (2019).

[58] I. T. Jolliffe, Principal Component Analysis, Springer Series in Statistics (Springer, New York, 2002).

[59] R. Dubessy, C. De Rossi, T. Badr, L. Longchambon, and H. Perrin, Imaging the Collective Excitations of an Ultracold Gas Using Statistical Correlations, New J. Phys. 16, 122001 (2014).
[60] I. Barr, Investigating the Dynamics of a Bose Einstein Condensate on an Atom Chip, Ph. D. thesis, Imperial College London, 2015, https://spiral.imperial.ac.uk/handle/10044/1/ 26226.

[61] Q. Zhou and T.-L. Ho, Universal Thermometry for Quantum Simulation, Phys. Rev. Lett. 106, 225301 (2011).

[62] T. Langen, R. Geiger, and J. Schmiedmayer, Ultracold Atoms out of Equilibrium, Annu. Rev. Condens. Matter Phys. 6, 201 (2015).

[63] J. Eisert, M. Friesdorf, and C. Gogolin, Quantum ManyBody Systems out of Equilibrium, Nat. Phys. 11, 124 (2015).

[64] A. Del Campo and W. H. Zurek, Universality of Phase Transition Dynamics: Topological Defects from Symmetry Breaking, Int. J. Mod. Phys. A 29, 1430018 (2014). 